\title{
Use Dependence of Presynaptic Tenacity
}

\author{
Arava Fisher-Lavie, ${ }^{1}$ Adel Zeidan, ${ }^{1}$ Michal Stern, ${ }^{1}$ Craig C. Garner, ${ }^{2}$ and Noam E. Ziv ${ }^{1}$ \\ ${ }^{1}$ Department of Physiology and Biophysics and Rappaport Institute, Technion Faculty of Medicine, and Network Biology Research Laboratories, Lorry \\ Lokey Center for Life Sciences and Engineering, Haifa 32000, Israel, and ${ }^{2}$ Department of Psychiatry and Behavioral Science, Nancy Pritzker Laboratory, \\ Stanford University, Palo Alto, California, 94304-5485
}

Recent studies indicate that synaptic vesicles (SVs) are continuously interchanged among nearby synapses at very significant rates. These dynamics and the lack of obvious barriers confining synaptic vesicles to specific synapses would seem to challenge the ability of synapses to maintain a constant amount of synaptic vesicles over prolonged time scales. Moreover, the extensive mobilization of synaptic vesicles associated with presynaptic activity might be expected to intensify this challenge. Here we examined the ability of individual presynaptic boutons of rat hippocampal neurons to maintain their synaptic vesicle content, and the degree to which this ability is affected by continuous activity. We found that the synaptic vesicle content of individual boutons belonging to the same axons gradually changed over several hours, and that these changes occurred independently of activity. Intermittent stimulation for $1 \mathrm{~h}$ accelerated rates of vesicle pool size change. Interestingly, however, following stimulation cessation, vesicle pool size change rates gradually converged with basal change rates. Over similar time scales, active zones (AZs) exhibited substantial remodeling; yet, unlike synaptic vesicles, AZ remodeling was not affected by the stimulation paradigms used here. These findings indicate that enhanced activity levels can increase synaptic vesicle redistribution among nearby synapses, but also highlight the presence of forces that act to restore particular set points in terms of SV contents, and support a role for active zones in preserving such set points. These findings also indicate, however, that neither AZ size nor SV content set points are particularly stable, questioning the long-term tenacity of presynaptic specializations.

\section{Introduction}

Presynaptic sites in the mammalian CNS often appear as small varicosities (presynaptic boutons) distributed along axons in an irregular, near-random fashion (Hellwig et al., 1994; Shepherd et al., 2002 and references within). Synaptic connections arranged in this form (en passant synapses) allow a single axon to contact multiple postsynaptic cells as it courses through the neuropil. Ultrastructural analysis reveals that synaptic boutons of en passant synapses typically lack obvious physical barriers that would act to separate presynaptic contents from the cytoplasm and membrane of the axon proper (Shepherd and Harris, 1998). The lack of such barriers would seem to challenge the ability of individual synapses to preserve their complement of presynaptic molecules and synaptic vesicles (SVs), in particular when considering the cellular and molecular dynamics associated with SV recycling, such as the dispersal of presynaptic molecules into the axoplasm and membrane of flanking axonal segments and their

\footnotetext{
Received July 4, 2011; revised Sept. 26, 2011; accepted Sept. 26, 2011.

Author contributions: A.F.-L., C.C.G., and N.E.Z. designed research; A.F.-L. and M.S. performed research; A.Z. and C.C.G. contributed unpublished reagents/analytic tools; A.F.-L., A.Z., M.S., and N.E.Z. analyzed data; A.F.-L. and N.E.Z. wrote the paper.

This work was supported by grants from United States Israel Binational Science Foundation (2007425) to C.C.G and N.E.Z, the German-Israeli Foundation for Scientific Research and Development (GIFI-840-194.1/2004) to N.E.Z., and NS39471 to C.C.G. The research leading to these results has received funding from the European Union Seventh Framework Programme under Grant Agreement HEALTH-F2-2009-241498 ("EUROSPIN" project). We are grateful to Larisa Goldfeld for her invaluable technical assistance.

The authors declare no competing financial interests.

Correspondence should be addressed to Dr. Noam E. Ziv, Network Biology Research Laboratories, Fishbach Building, Technion City, Haifa 32000, Israel. E-mail noamz@netvision.net.il.

DOI:10.1523/JNEUROSCI.3384-11.2011

Copyright $\odot 2011$ the authors $\quad 0270-6474 / 11 / 3116770-11 \$ 15.00 / 0$
}

subsequent redistribution among neighboring boutons (Sankaranarayanan and Ryan, 2000; Chi et al., 2001, 2003; Colicos et al., 2001; Li and Murthy, 2001; Krueger et al., 2003; Star et al., 2005; Tao-Cheng et al., 2006; Tsuriel et al., 2006).

In addition to presynaptic molecules, SVs seem to be continuously interchanged among presynaptic sites along the same axons, where they become incorporated into local vesicles pools and function as native vesicles (Krueger et al., 2003; Darcy et al., 2006; Fernandez-Alfonso et al., 2008; Staras et al., 2010) (see also Kraszewski et al., 1995; Dai and Peng, 1996; Ahmari et al., 2000; Hopf et al., 2002; De Paola et al., 2003; Westphal et al., 2008) (for reviews, see Staras, 2007; Staras and Branco, 2010). This interchange, in which individual vesicles or small packets of SVs migrate from one bouton to another, effectively forms a "superpool" of vesicles shared by neighboring boutons (Westphal et al., 2008; Staras et al., 2010). Importantly, exchange rates can be surprisingly high (estimated to be $\sim 4 \%$ per minute), and thus migrating vesicles can constitute a substantial fraction (estimated at $\sim \geq 40 \%$ ) (Staras et al., 2010; Westphal et al., 2008) of the SV pool available for secretion.

The significant rates at which SVs and vesicle packets migrate between synapses raise questions concerning the ability of individual presynaptic boutons to preserve their respective SV contents over long durations. How tenacious are individual boutons in this respect? Moreover, the extensive mobilization of SVs associated with activity-evoked exocytosis would seem to further challenge this ability. If so, to what degree does intense synaptic activity affect this ability?

Here we describe experiments aimed at examining the stability of total SV pool sizes at individual synapses and at determining 
how total SV pool size stability is affected by prolonged periods of synaptic activity.

\section{Materials and Methods}

Cell culture. Hippocampal cell cultures were prepared from 1- to 2-d-old Sprague Dawley rats (of either sex) and grown in $8 \mathrm{~mm}$ glass cloning cylinders as described previously (Tsuriel et al., 2006). Neurons were used for experiments 16-24 d after plating. Mice cortical neuron cultures were prepared from 1-d-old Munc13-1:enhanced yellow fluorescent protein (EYFP) knock-in mutant mice (Kalla et al., 2006). Reagents were procured from the following sources: CNQX (6-cyano-7-nitroquinoxaline-2,3-dione) from Tocris Bioscience; AP-5 (2-amino-5-phosphonopentanoic acid) from Sigma; and FM4-64 (N-(3-triethylammoniumpropyl)-4-(p-dibutylaminostyryl)pyridinium, dibromide) from Invitrogen.

DNA construct, virus production, and transduction. EGFP:SV2A, a fusion protein of EGFP and rat SV2A (GenBank no. L01788.1) was introduced into neurons by means of a third-generation lentiviral expression system based on an FUGW backbone (Lois et al., 2002). HEK293T cells were cotransfected with a mixture of FU-GFP:SV2A (Lucido et al., 2009) and three packaging plasmids: pLP1, pLP2, and pLP\VSVG (Invitrogen). Transfection was performed when the cells had reached $80 \%$ confluence, and supernatant was collected after $48 \mathrm{~h}$, filtered through $0.45 \mu \mathrm{m}$ filters, aliquoted, and stored at $-70^{\circ} \mathrm{C}$. Transduction of hippocampal cultures was performed on day 2 in vitro by adding $1-2 \mu$ of the filtered supernatant to each cloning cylinder. The expression of GFP:Bsn95-3938 (a fusion protein of GFP and a slightly truncated variant of Bassoon) (Dresbach et al., 2003; Bresler et al., 2004; Tsuriel et al., 2009) was performed by calcium phosphate transfection on days 7-8 in vitro, as described previously (Bresler et al., 2004).

Long-term imaging. Neuronal cultures were mounted on a custombuilt confocal laser scanning microscope that was controlled by software written by one of us (N.E.Z.) and includes provisions for automated, multisite time-lapse microscopy (Tsuriel et al., 2006). Fluorescence and differential interference contrast images were acquired using a $40 \times, 1.3$ numerical aperture Fluar objective. EGFP, and FM4-64 were excited by using the $488 \mathrm{~nm}$ line of an argon laser (JDS Uniphase), whereas EYFP was excited using the $514 \mathrm{~nm}$ line of the same laser. Fluorescence emissions were read using 500-545 $\mathrm{nm}$ bandpass, >630 nm long-pass, and 520-570 bandpass filters, respectively (Chroma). Long-term experiments not requiring stimulation were performed without perfusion, within the intact cloning cylinder. In the experiments involving stimulation protocols (including nonstimulated controls), the cells were mounted in a custom-built chamber and continuously perfused using an ultraslow peristaltic pump ( $\sim 5 \mathrm{ml} / \mathrm{d})$ with feeding medium: Minimal Essential Medium (Sigma), $25 \mathrm{mg} / \mathrm{L}$ insulin (Sigma), $20 \mathrm{~mm}$ glucose (Sigma), 1 mM L-glutamine (Sigma), $5 \mu \mathrm{g} / \mathrm{ml}$ gentamycin sulfate (Sigma), and 2\% B-27 supplement (Invitrogen). Unless otherwise specified, the medium also included CNQX $(10 \mu \mathrm{M})$ and AP-5 (50 $\mu \mathrm{M})$. A mixture of 95\% air with 5\% $\mathrm{CO}_{2}$ was continuously streamed into a custom-built enclosure placed over the cells. The chamber base and objective were heated to 36 and $35^{\circ} \mathrm{C}$, respectively, using resistive elements, separate temperature sensors, and controllers, resulting in temperatures of $\sim 35^{\circ} \mathrm{C}$ in the culture medium.

Time-lapse recordings were performed by averaging five frames at three to four focal planes spaced $0.8 \mu \mathrm{m}$ apart. All data were collected at a resolution of $640 \times 480$ pixels, at 12 bits/pixel. Data were collected sequentially from up to seven predefined sites (fields of view), using a robotic $X Y Z$ stage to cycle automatically through these sites. Focal drift was corrected before collecting each image stack using the "autofocus" feature of the microscope system. Stimulation was performed by passing $1 \mathrm{~ms}, 18 \mathrm{~mA}$ current pulses from an isolated stimulator (World Precision Instruments) through platinum electrodes placed on both sides of the chamber. Intermittent stimulation for $1 \mathrm{~h}$ periods was timed using a stimulus generator (World Precision Instruments) slaved to the confocal microscope. FM4-64 labeling was performed as follows: the perfusion chamber was flooded with Tyrodes solution $(119 \mathrm{~mm} \mathrm{NaCl}, 2.5 \mathrm{~mm} \mathrm{KCl}$, $2 \mathrm{mM} \mathrm{CaCl}_{2}, 2 \mathrm{~mm} \mathrm{MgCl}_{2}, 25 \mathrm{~mm}$ HEPES, $30 \mathrm{~mm}$ glucose, buffered to $\mathrm{pH}$ 7.4) containing $15 \mu \mathrm{M}$ FM 4-64, stimulated for $60 \mathrm{~s}$ at $10 \mathrm{~Hz}$, left in the dye for an additional $30 \mathrm{~s}$, washed for $1 \mathrm{~min}$ with $1 \mathrm{~mm}$ ADVASEP 7 ( $\beta$-cyclodextrin sulfbutyl ether, cydex) in Tyrodes solution, and then washed rapidly with Tyrodes solution for an additional $60 \mathrm{~s}$. Dye unloading was performed by stimulating the neurons for $120 \mathrm{~s}$ at $10 \mathrm{~Hz}$.

Image analysis. All data analysis was performed using software (OpenView) specially written for this purpose by one of us (N.E.Z.) on maximal intensity projections of $Z$-section stacks. Intensities of fluorescent puncta were measured as mean pixel values in $7 \times 7$ pixel $(\sim 1 \times 1 \mu \mathrm{m})$ rectangular regions centered on individual puncta. These were recentered on the same puncta in the entire time series using automatic tracking algorithms implemented in OpenView. All tracking was verified and, if necessary, manually corrected. In a typical experiment, $\sim 3-5 \%$ of the puncta initially selected for analysis disappeared over a $3 \mathrm{~h}$ time course, and another $10-20 \%$ were rejected due to tracking uncertainties (puncta splitting or merging with neighboring puncta). Only data from puncta tracked successfully for the entire duration of experiment were included here. A shuffled data similarity index (SI) calculation was performed by cyclically permutating the last time point measurement vector of puncta fluorescence values and calculating the SI of the shuffled data against the unshuffled $t=0$ fluorescence value vector. This was repeated 10 times for each site, and the average shuffled data SI was calculated. For kymograph analysis, dedicated software was developed by one of us (A.Z.). Lines were drawn manually along axonal segments, and the fluorescence profile along the line was obtained (each point along the line representing the average fluorescence of a perpendicular line with a width of $0.75 \mu \mathrm{m}$ ). The line was updated for each consecutive image in the time series, and the profile for each image was collected. Data analysis was performed using Matlab (Mathworks) and Microsoft Excel. Final images were prepared using Adobe Photoshop and Microsoft PowerPoint.

\section{Results \\ Following long-term changes in the SV content of individual synapses}

The ability of a presynaptic bouton to support sustained neurotransmitter release depends to a large degree on the size of its vesicle pool (Murthy et al., 1997; Waters and Smith, 2002). However, recent studies, such as those described above, indicate that the ability of any particular presynaptic site to maintain its pool of vesicles is constantly challenged by activity-dependent and -independent processes. To assess the ability of individual presynaptic boutons to preserve the total size of their vesicle pools over long durations, we tagged SVs with a fluorescent marker, recorded long-term changes in the sizes of vesicle pools at individual boutons formed along axons of hippocampus neurons in primary culture, and followed the redistribution of SVs among such boutons.

To obtain measures of total SV pool sizes at individual synapses, we expressed an EGFP-tagged variant of the synaptic vesicle protein SV2A (EGFP:SV2A) and measured changes in EGFP: SV2A fluorescence. SV2A (Bajjalieh et al., 1992; Feany et al., 1992) is a conserved, highly glycosylated integral membrane protein whose function is not well understood but has been linked to the regulation of neurotransmitter release (Nowack et al., 2010 and references therein). Importantly, SV2A is specifically localized to SVs and is therefore a useful tag for following the dynamics of these organelles.

EGFP:SV2A was expressed in a small number of neurons by means of a third-generation lentiviral expression system (Lois et al., 2002; Lucido et al., 2009) (see also Nowack et al., 2010) as we previously found that this method leads to very modest overexpression levels (Minerbi et al., 2009). When expressed in cultured neurons, EGFP:SV2A exhibits a punctate distribution pattern (Fig. 1A). Due to the very sparse infection, most EGFP:SV2A puncta within a field of view belonged to a very small number of presynaptic neurons, only one in most cases. EGFP:SV2A puncta localized nearly perfectly with functional presynaptic sites as measured by stimulation-evoked uptake of the styryl dye FM4-64 

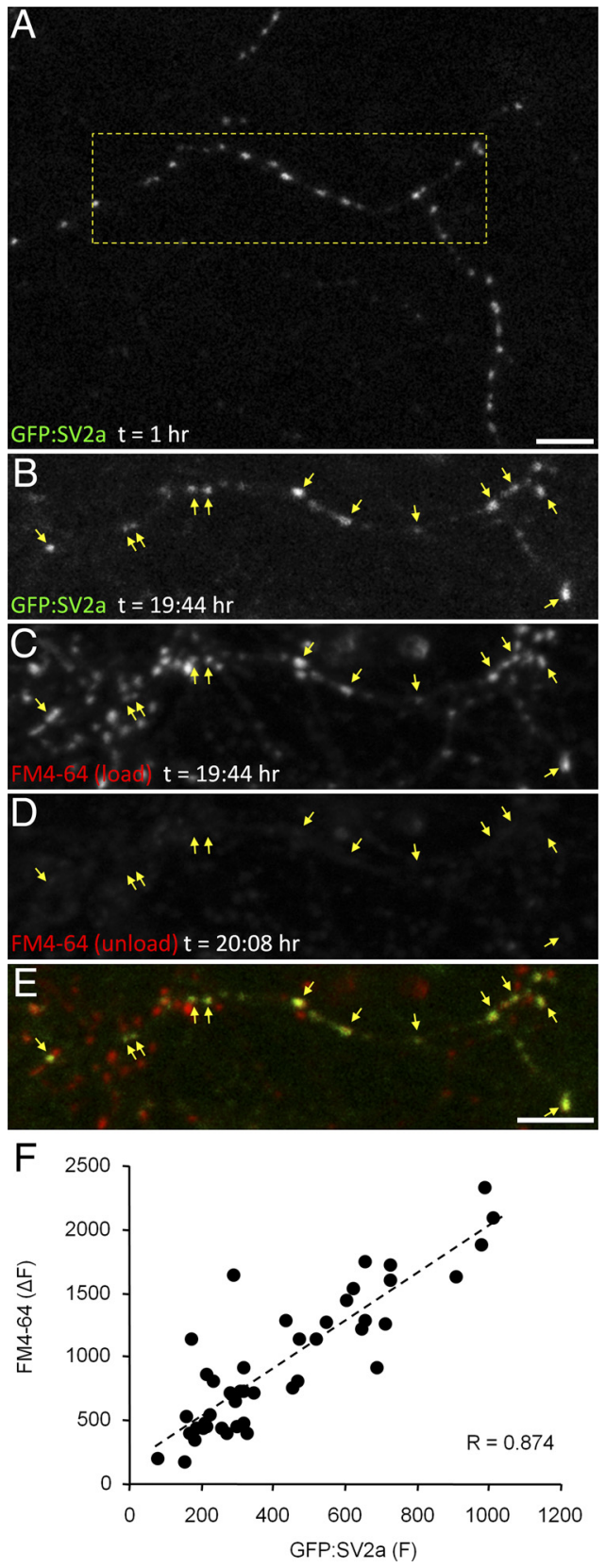

Figure 1. Expression of EGFP:SV2A in cultured hippocampal neurons. $A$, A fluorescence image of an axon belonging to a neuron expressing EGFP:SV2A. $\boldsymbol{B}$, A higher magnification of the same region shown in $\boldsymbol{A}$ at the end of a $\sim 20 \mathrm{~h}$ time-lapse session. $\boldsymbol{C}$, Same region as in $\boldsymbol{B}$ after labeling with FM4-64 by field stimulation for $1 \mathrm{~min}$ at $10 \mathrm{~Hz}$. Note the good spatial correspondence between EGFP:SV2A and FM4-64-labeled puncta (yellow arrows). $\boldsymbol{D}$, Same region as in $\boldsymbol{B}$ after unloading the FM4-64 (2 min at $10 \mathrm{~Hz}$ ). $\boldsymbol{E}$, Correlation between EGFP:SV2A fluorescence and FM4-64 fluorescence for the same region. Scale bars, $10 \mu \mathrm{m}$.

(Fig. $1 B-E$ ). Quantitative analysis revealed a good correspondence between EGFP:SV2A puncta fluorescence and FM4-64 uptake (Fig. $1 F$ ) ( 4 experiments, 135 boutons, $R=0.87,0.92,0.64$, and 0.82). Interestingly, FM4-64 uptake was no larger in EGFP: SV2A-positive puncta compared with presynaptic boutons of neighboring uninfected axons (EGFP:SV2A-positive/all boutons $=1.03 \pm 0.14 ; n=135$ and $n=819$, respectively). These observations suggest that EGFP:SV2A fluorescence can be used as an estimate of total SV pool size at individual boutons and, more importantly, to measure how sizes of such pools change over time.
To examine how stable total SV pool sizes remain over long durations, neurons expressing EGFP:SV2A were placed on a custombuilt confocal laser scanning microscope, and maintained in a sterile atmospheric environment of $5 \% \mathrm{CO}_{2}, 95 \%$ air at $35^{\circ} \mathrm{C}$. The hippocampal cultures were kept in growth medium that was slowly circulated by an ultraslow perfusion system to improve the longterm preparation vitality (Minerbi et al., 2009). Images were collected at a rate of one image every $5 \mathrm{~min}$ for durations of $\geq 3 \mathrm{~h}$. To minimize the effects of spontaneous activity, the glutamate receptor blockers AP-5 $(50 \mu \mathrm{M})$ and CNQX $(10 \mu \mathrm{M})$ were added to the external solution (Wagenaar et al., 2006; Gal et al., 2010).

To quantify the stability of SV cluster sizes at individual presynaptic sites, we tracked individual EGFP:SV2A puncta in the image time series (see Materials and Methods for details) and quantified the fluorescence of individual EGFP:SV2A puncta over time (Fig. 2A-C). As previously described (De Paola et al., 2003; Krueger et al., 2003; Darcy et al., 2006; Staras et al., 2010), SV clusters often exhibited significant dynamics over time scales of several minutes (merging, splitting, and lateral movements). All subsequent analysis was therefore limited to EGFP:SV2A puncta that could be tracked reliably throughout the experiments.

Plotting the fluorescence profiles for individual puncta over periods of several hours (Fig. $2 A, B$, three marked puncta) indicates that the SV cluster size of individual boutons can exhibit significant changes over time, presumably reflecting SV redistribution among neighboring synapses (Darcy et al., 2006; Staras et al., 2010). To quantify the extent of such changes, we measured, for each bouton, the normalized range of EGFP:SV2A fluorescence change, allowing us to compare changes in boutons with widely different absolute fluorescence values. This measure was calculated, as illustrated in Figure $2 D$, according to the following equation:

$$
\frac{\text { Range }}{\text { mean }}=100 \times\left(F_{\max }-F_{\min }\right) / \bar{F}
$$

where $F_{\max }$ is the maximal fluorescence measured for a given bouton during the experiment, $F_{\min }$ is the minimal fluorescence, and $\bar{F}$ is the mean fluorescence value for the same period. To minimize the effects of measurement noise, the values were calculated after "smoothing" the data with a 5-point low-pass filter (Fig. 2C,D).

The distribution of normalized ranges for all cells followed in the experiment of Figure 2 ( 5 cells, 79 boutons) is shown in Fig. $2 \mathrm{E}$. The average normalized range of fluorescence change in this experiment $(>8 \mathrm{~h}$ ) was $46 \%$, with many boutons exhibiting significantly greater changes ( $>80 \%$ in extreme cases). These observations indicate that the SV contents of individual boutons can change substantially over relatively short periods.

To quantify the degree to which neighboring EGFP:SV2A puncta changed their sizes relative to each other, we applied two measures. The first — relative remodeling (Minerbi et al., 2009) quantifies changes in the rank order of puncta over time. The basic idea was to sort synapses according to their sizes or, more specifically, sort puncta according to their fluorescence. Then, at each subsequent time point, sort the puncta again according to their new fluorescence values. The degree to which each synapse changed its rank relative its original rank was then determined, and finally, all rank changes for all synapses were summed and normalized to give a value between 0 and 1 . This measure, $M_{t}$, is given as follows:

$$
M_{t}=\frac{2}{n^{2}} \sum_{i=1}^{n}\left|r_{i_{t}}-r_{i_{0}}\right|
$$



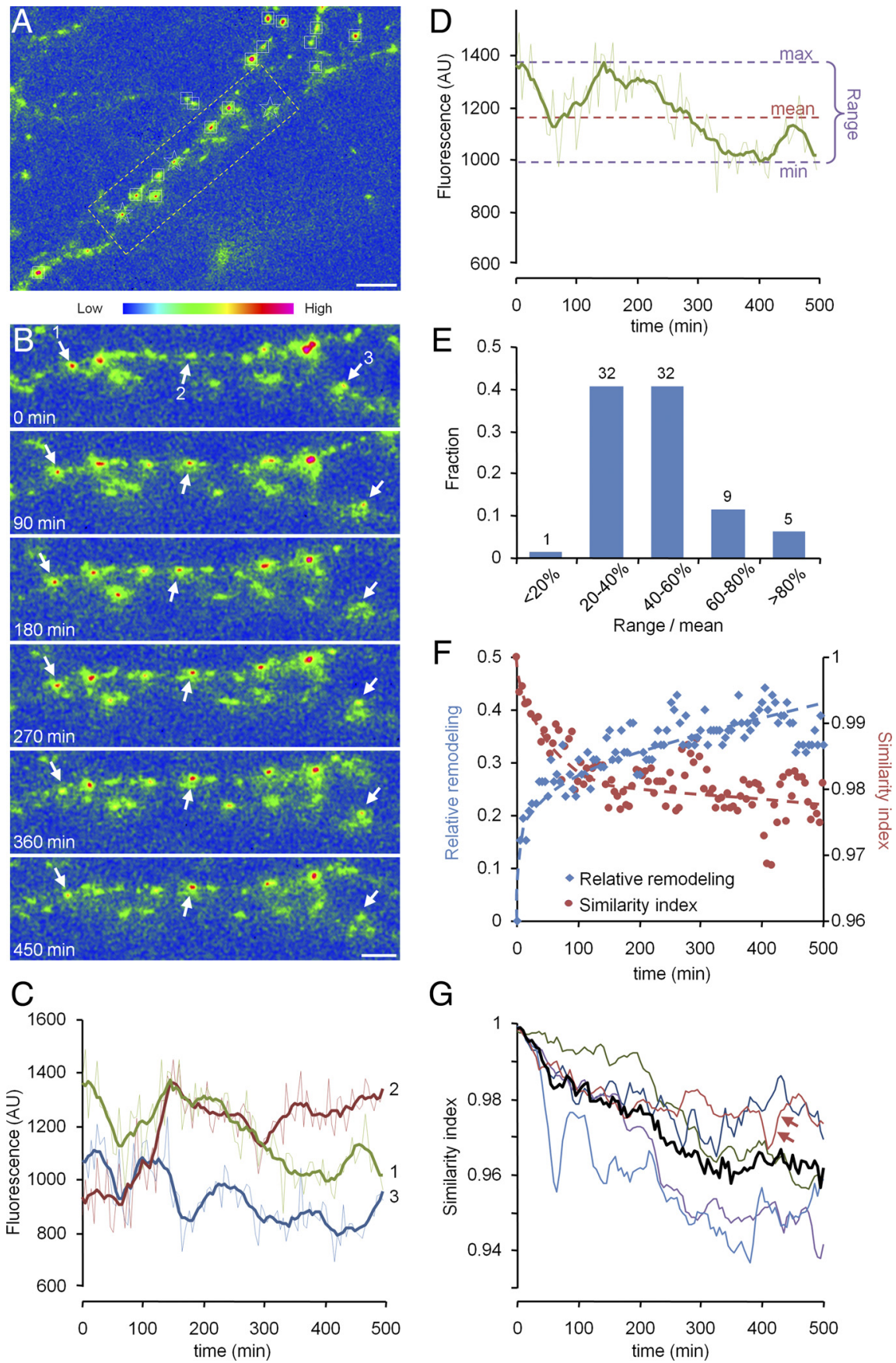

G

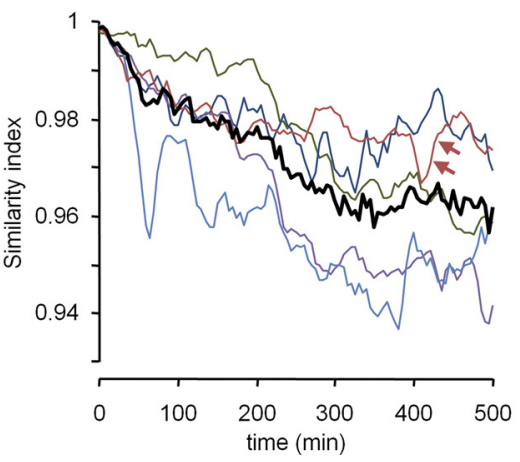

Figure 2. Long-term changes in EGFP:SV2A fluorescence. $A$, An axon belonging to a neuron expressing EGFP:SV2A. Fluorescence levels are encoded in false color according to color scale at bottom of the panel. Scale bar, $10 \mu \mathrm{m}$. $\boldsymbol{B}$, Time-lapse image of region enclosed in rectangle in $A$. Images were collected at 5 min intervals (only a subset of these shown here) for $\sim 500 \mathrm{~min}$. Scale bar, $5 \mu \mathrm{m}$. $C$, Changes in fluorescence over time for three boutons pointed to by arrows in $\boldsymbol{B}$, stars in $\boldsymbol{A}$. Raw measurements depicted by thin lines; smoothed (7-point low-pass filter) depicted as thick lines. $\boldsymbol{D}$, Illustration of the range/mean (normalized range) measure calculated here for bouton 1 in $A-C$. $E$, Distribution of normalized range values for all boutons in this experiment ( 5 fields of view, 89 boutons). $\boldsymbol{F}$, Measures of changes in relative sizes of SV pools. Two measures were calculated for all boutons enclosed in rectangles/stars in $\boldsymbol{A}$ : relative remodeling (Minerbi et al., 2009; blue) and SI (Sasaki et al., 2007; red). Note the gradual and symmetrical decay of both measures. $\mathbf{G}$, SI decay rates for all five cells followed in this experiment. The thick black line is the average $\mathrm{SI}$ decay rate. The line pointed to by arrows is the same data shown in $\boldsymbol{F}$.

where $n$ is the population of tracked EGFP:SV2A puncta, $r_{i t}$ is the rank of bouton $i$ at time $t$, and $r_{i 0}$ its rank at time $t=0$. $M_{t}$ will approach 1 if the rank of each synapse at time $t$ is furthest away from its rank at time $t=0$, and will approach 0.67 if the ranks at time $t$ bear no relationships beyond chance. As shown in Figure 2F, the value of $M_{t}$ increases significantly over time even under baseline conditions in which spontaneous activity was suppressed.

$M_{t}$ is based on ranking and is therefore does not ideally capture quantitative changes. We therefore also quantified SV redistribution using a second measure, the SI (Sasaki et al., 2007), as follows:

$$
S I_{t}=\frac{\vec{g}_{0} \cdot \vec{g}_{t}}{\left\|\vec{g}_{0}\right\| \cdot\left\|\vec{g}_{t}\right\|}
$$

where $\vec{g}_{0}$ is a vector of fluorescence intensities at time $t=0$, and $\vec{g}_{t}$ describes the fluorescence of the same puncta at time $t$. As $\mathrm{SI}_{\mathrm{t}}$ is essentially the cosine of the angle between the two vectors, a value of 1 means no change in size distribution (an angle of 0 ); increasingly smaller values indicate increasingly reduced similarity between the two vectors, in practice meaning a greater degree of fluorescence redistribution. To minimize the effects of measurement noise on the reference vector $g_{0}$, its values were set to be the average fluorescence intensities of each bouton in the first three time points (hence, SI is not exactly 1 at $t=0$ ). Note that SI is not affected by multiplication of $g_{t}$ with a scalar (which only affects the vectors length, not its angle) and thus should not be affected by photobleaching (which can be approximated by multiplication of all fluorescence values by a constant $<1.0$ ). As shown in Figure $2 F, \mathrm{SI}_{t}$ decays gradually over time, in a manner that is qualitatively similar and symmetrical to the gradual change in $M_{t}$. Although both $M_{t}$ and $\mathrm{SI}_{t}$ show symmetrical, qualitatively similar trends (Fig. $2 F$ ), only SI will be used hereafter as it better captures quantitative changes.

The SI decay rate for all five cells followed in this experiment is shown in Figure $2 G$. Note that the SI decayed, on average, by only $4 \%$ over this duration. Compared with the large fluctuations revealed by the normalized range analysis described above (Fig. 2E), this value appears at first sight to be surprisingly small. This apparently small value, however, is actually very substantial: the average SI calculated for shuffled data (see Materials and Methods for details) resulted in an SI value of $\sim 0.89$ at the end of the experiment. This implies that the full dynamic range of this measure in these experiments is from 1.0 (no change) to $\sim 0.89$ (random fluorescence values). It follows that an SI decay of $4 \%$ of $11 \%$ is indicative of extensive SV redistribution. It should be noted that the shuffled SI value depends solely on the range and distribution of fluorescence values measured during an experiment, not on the dynamics or stability of 
EGFP:SV2A puncta fluorescence values. Thus, the shuffled SI value represents the minimum attainable SI value in an imaginary situation in which the fluorescence of each particular synapse changes to that of some other synapse selected randomly from the EGFP:SV2A puncta population. Interestingly, despite large fluctuations of EGFP:SV2A fluorescence at individual boutons (Fig. 2E) SI values tended to plateau above the value of 0.89 , which indicates that SVs do not redistribute in a completely random fashion among neighboring boutons. This may be taken to suggest that individual boutons are capable of maintaining their SV pool sizes within some range, at least over time scales of several hours.

As mentioned above, only EGFP:SV2A puncta that could be identified and tracked reliably were included in this type of analysis. To maximize the information and include the dynamics of mobile SV packets that travel along axons between more stable puncta, and to reduce the bias toward stable objects, we developed software for kymograph analysis of entire axonal segments (Fig. 3A). This analysis is based on profiling the fluorescence intensity along axonal segments, and quantifying changes in such profiles over time by correlating (Pearson's correlation function) the profiles at time $t$ and the profile at $t=0$ (Fig. $3 B$ ) (see Materials and Methods for further details). As shown in Figure $3 C$, the outcome of this analysis is rather noisy, but the trend of reduced correlation with time is clearly observable, in particular when averaging correlation values over half-hour (six-time point) bins.

\section{Stimulation leads to the partial dispersion of EGFP:SV2A puncta}

Previous studies have shown that synaptic activation is associated with the dispersal of presynaptic molecules into the axoplasm and membrane of flanking axonal segments and their subsequent redistribution among neighboring boutons (Chi et al., 2001, 2003; Li and Murthy, 2001; Star et al., 2005; Fernandez-Alfonso et al., 2006; Tsuriel et al., 2006; Wienisch and Klingauf, 2006). To examine whether EGFP:SV2A exhibits a similar response to stimulation, we followed changes in EGFP:SV2A distribution following electrical stimulation. To that end, we stimulated neurons expressing EGFP:SV2A at $20 \mathrm{~Hz}$ for $30 \mathrm{~s}$, and collected images before, during, and after the stimulation period at $30 \mathrm{~s}$ intervals. As mentioned above, stimuli were performed in AP-5 and CNQX to minimize spontaneous activity and stimuli-induced reverberations. As shown in Figure $4 A$, stimulation led to significant dispersion of EGFP:SV2A puncta, which was, in some cases, associated with observable changes in bouton morphology (Fig. $4 \mathrm{~B}$ ). Following stimulation cessation, EGFP:SV2A fluorescence recovered gradually over a time course of a few minutes. The fluorescence recovery phase could be fit to a weighted $(\sim 1: 2)$ sum of two exponentials with time constants of $\sim 0.6$ and $8.6 \mathrm{~min}$, respectively (Fig. 4C) (5 separate experiments, 318 boutons).

On average, stimulation led to reduction of $\sim 12 \%$ of puncta fluorescence. This value, however, was quite variable and differed over a wide range from one synapse to another (Fig. $4 D$ ), ranging from a loss of $\sim 50 \%$ in some synapses to a gain of $\sim 20 \%$ at others (apparently reflecting the temporary mobilization of EGFP:SV2A from neighboring synapses). No consistent relationship between puncta size and the degree of dispersion was found (data not shown).

We do not know whether activity-induced dispersion of EGFP:SV2A reflects the insertion of SV2A into the axonal membrane followed by lateral diffusion (Li and Murthy, 2001; Fernández-Alfonso et al., 2006; Wienisch and Klingauf, 2006) or
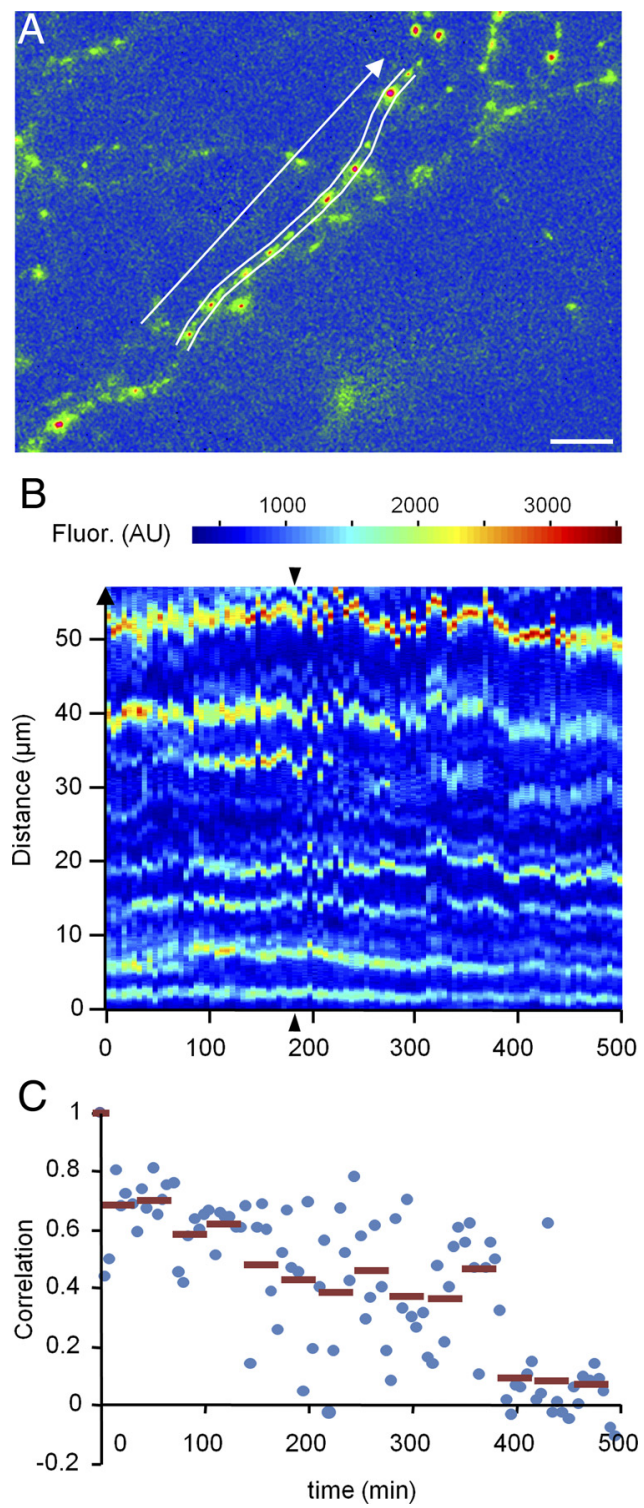

Figure 3. A, Kymograph analysis of the same axonal segment as in Figure $2 A$. Lines depict the region used to obtain the fluorescence profile along this axon. Arrow shows direction of profile vector. $t=180 \mathrm{~min}$. $\boldsymbol{B}$, Changes in fluorescence profiles over time. Fluorescence is encoded in color according to scale bar above graph. Profile vector direction and distance along vector are shown on the left-hand side. Arrowheads point to the profile of the time point shown in $\boldsymbol{A}$. Note the jagged appearance of the color stripes indicating changes in the longitudinal position of fluorescent puncta. $\boldsymbol{C}$, Correlation (Pearson) of profile vectors shown in $\boldsymbol{B}$ with vector at time $t=0$ for all consecutive time points (blue circles). Red horizontal lines are averages of correlation values over 30 min intervals.

the dispersal of intact SVs. However, these findings show that in common with other integral (VAMP2; Li and Murthy, 2001) and SV-associated proteins (rab3, Synapsin) (Chi et al., 2001, 2003; Star et al., 2005), evoked presynaptic activation leads to robust EGFP:SV2A mobilization.

\section{Relationships between synaptic activity and changes in total SV pool sizes of individual synapses}

As described above, SVs are continuously exchanged among neighboring synapses, forming what has been referred to as a SV superpool. Given that evoked activity causes extensive SV mobilization, we wondered whether increased SV mobilization would accelerate changes in the relative sizes of SV pools within popu- 

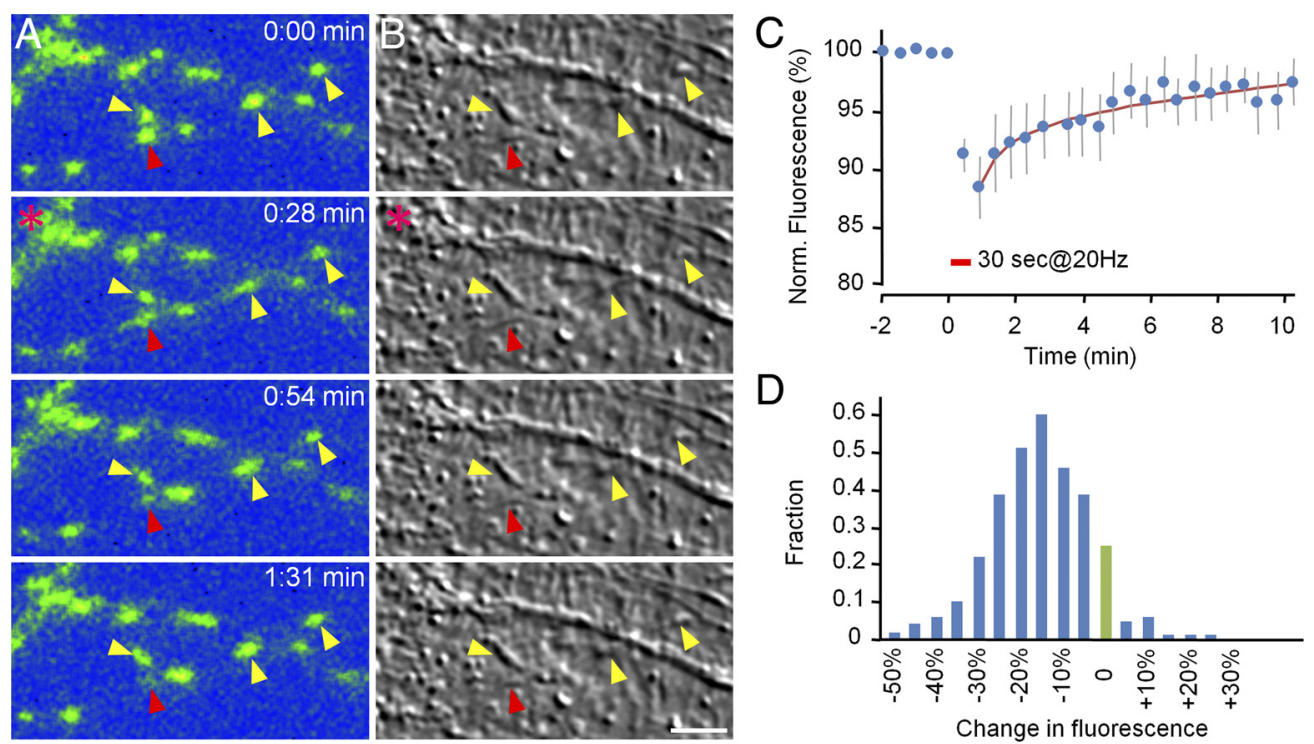

Figure 4. Stimulation leads to a temporary dispersion of EGFP:SVZA fluorescence. A, An axon belonging to a neuron expressing EGFP:SV2A. Fluorescence levels are encoded in false color according to color scale in Figure $2 \mathrm{~A}$. A 30 s stimulation at $20 \mathrm{~Hz}$ is followed by the dispersion of EGFP:SV2A fluorescence that recovers gradually over the next few minutes. The panels annotated with asterisks were collected $\sim 15 \mathrm{~s}$ after the beginning of the stimulation train. $\boldsymbol{B}$, In some cases, morphological changes in synaptic boutons were observable in differential interference contrast images of the same region (arrowheads). In very rare cases, EGFP:SV2A fluorescence failed to recover (red arrowhead). Scale bar, $5 \mu \mathrm{m}$. C, Average dispersion and recovery kinetics ( 5 experiments, 318 boutons; average \pm SEM). Red lines are fits to a sum of two exponentials with time constants of 0.6 and $8.6 \mathrm{~min}$. $\boldsymbol{D}$, Distribution of peak dispersion values (expressed as percentage of original bouton fluorescence). Negative values indicate fluorescence losses, while positive values indicate fluorescence gains. Note the large variability of these values. Same dataset as $\boldsymbol{C}$.

lations of synapses belonging to the same neurons. We thus performed the following experiments: Neurons expressing EGFP: SV2A were mounted as described above, and baseline images were collected at $5 \mathrm{~min}$ intervals for $40 \mathrm{~min}$ at four to seven predefined sites (fields of view), using a robotic $X Y Z$ stage to cycle automatically through these sites. Following this period, the neurons were stimulated repeatedly at $5 \mathrm{~min}$ intervals for $60 \mathrm{~min}$, each episode consisting of a $20 \mathrm{~Hz}$ train of $1 \mathrm{~ms}$ stimuli for $30 \mathrm{~s}$. The experiment ended with another hour of imaging with no further stimulation. Paired controls were performed, in which no stimulation was delivered. To minimize potential activityinduced changes in synaptic properties, the experiments were performed in the presence of AP-5 and CNQX.

Individual presynaptic puncta were tracked as described above, and the fluorescence of these puncta was measured at all time points. As shown in Figure $5 A$, the stimulation period was associated with a reduction in puncta fluorescence, due to activity-evoked dispersion of EGFP:SV2A. Importantly, however, EGFP:SV2A fluorescence recovered to control levels within $\sim 10$ min once the stimulation was stopped.

Changes in the relative sizes of SV pools were calculated using the SI measure described above. As shown in Figure $5 B$, stimulation increased the rate of SI decay, indicating that activity-induced SV mobilization accelerated the rate at which SV pool sizes of individual synapses changed in relation to each other. Interestingly, unlike the rapid rebound of fluorescence intensities following stimulation cessation, the SI did not exhibit an immediate rebound. Instead, it remained rather constant, gradually converging with the SI values recorded in nonstimulated preparations, and completely converging with these after $\sim 1 \mathrm{~h}$ from stimulation cessation. It is important to note that the SI in the paired controls did not remain constant either. In fact, SI in these preparations decayed monotonically over the entire experiment, pointing to the presence of activity-independent processes that lead to changes in the relative sizes of SV pools in neighboring presynaptic boutons (Fig. 2G).
The effects of stimulation on SV redistribution were also analyzed by kymograph analysis of entire axonal segments as described above and in Figure 3. Here, however, we did not observe a major effect of the $1 \mathrm{~h}$ stimulation period on the distribution rate (data not shown). Our inability to detect an effect could result from the rather noisy nature of this measure. Alternatively, however, it might suggest that the major features quantified by this measure, namely, small longitudinal shifts in synaptic position and mobility of vesicle packets are not significantly affected by increased activity levels, at least not at the time scales examined here.

We also performed similar experiments in which AP-5 and CNQX were omitted (Fig. 5A,B). Somewhat surprisingly, this omission did not have major effects on fluorescence intensity changes or SI decay rates compared with preparations stimulated in their presence.

Examining the normalized ranges of GFP:SV2A fluorescence changes (as in Fig. 2D,E) shows that GFP:SV2A fluorescence changed considerably in most synapses over this $\sim 3 \mathrm{~h}$ period (although to a lesser extent, which might be expected given the shorter duration of these experiments) (see also Fig. 7C), with $>59$ and $>13 \%$ of boutons exhibiting changes of $>20$ and $40 \%$, respectively (Fig. 5C). This analysis indicates that SI decay rates represent the synaptic population, rather than a small number of highly variable boutons (outliers). Furthermore, they indicate, as mentioned above, that the apparently modest drop in the SI $(\sim 1.5 \%$ in these experiments) is actually indicative of a significant redistribution of SVs among nearby boutons. Note that as stimulation-driven dispersion would affect normalized range values, this analysis was performed only for the nonstimulated dataset.

These findings suggest that increased activity levels and the SV mobilization they entail, somewhat accelerate changes in the relative sizes of SV pools within the populations of synapses belonging to the same axons. They also point, however, to the presence 

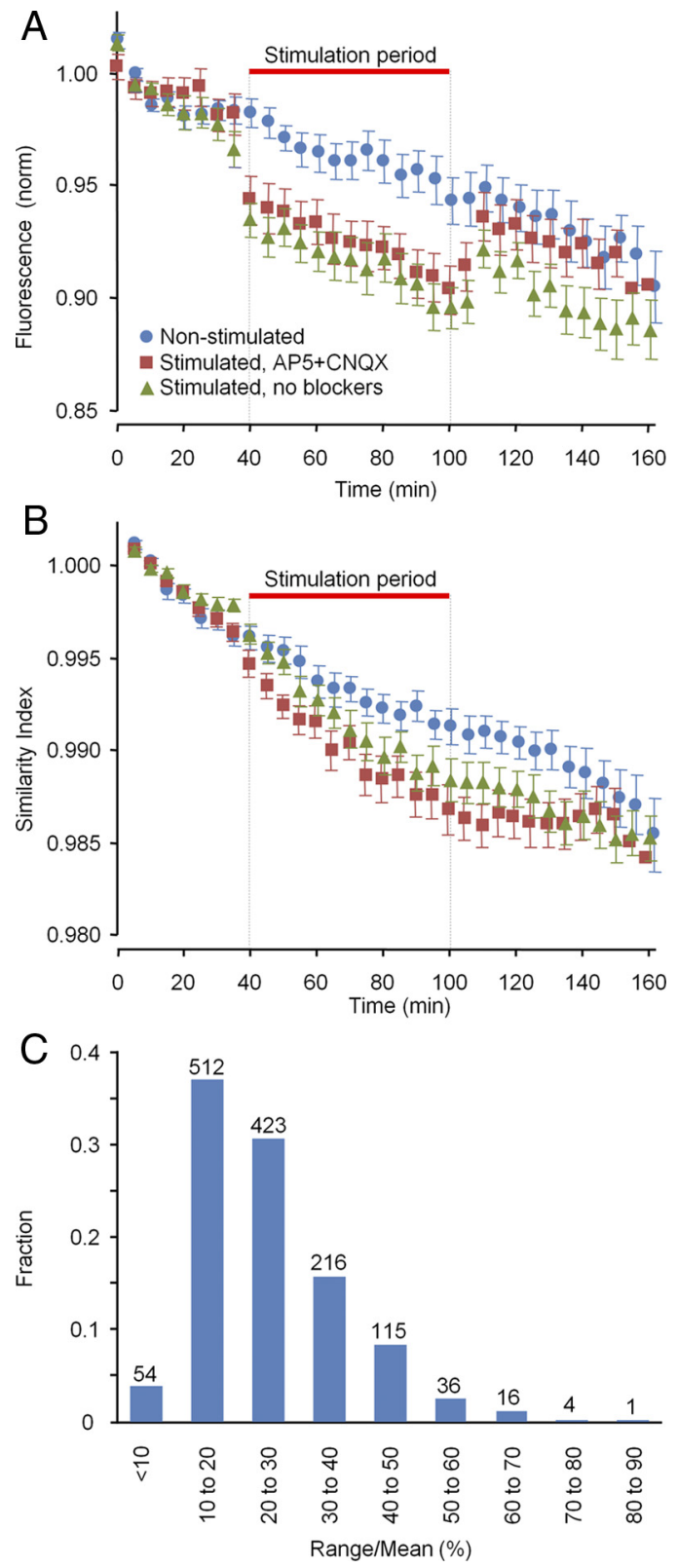

Figure 5. Use dependence of synaptic vesicle pool size tenacity. $\boldsymbol{A}$, Changes in EGFP: SV2A fluorescence over time before, during, and after a stimulation period ( $30 \mathrm{~s}$ at $20 \mathrm{~Hz}$ repeated every $5 \mathrm{~min}$ for $1 \mathrm{~h}$ ). Data are shown for three conditions (average $\pm \mathrm{SEM}$ ): no stimulation, in the presence of CNQX and AP5 (6 separate experiments, 25 sites, 1380 boutons); stimulation in CNQX and AP5 ( 6 separate experiments, 25 sites, 1308 boutons); and stimulation without CNQX and AP5 ( 4 separate experiments, 20 sites, 1132 boutons). In all conditions, stimulation led to a reduction of EGFP:SV2A fluorescence that recovered within $\sim 10 \mathrm{~min}$ of stimulation cessation. The gradual reduction in baseline fluorescence $(\sim 10 \%$ over $3 \mathrm{~h})$ is due to photobleaching. Note that due to the sequential multisite imaging procedure, the temporal relationships between stimulus initialization/termination and imaging timing differed slightly between sites. As a result, the average dispersion-related fluorescence loss appears to be reduced compared with the timelocked, high temporal resolution experiments of Figure 4. B, SI decay rates for the same data and conditions as in $\boldsymbol{A}$ (average $\pm \mathrm{SEM}$ ). Under baseline conditions, the SI decays gradually (this, however, is not due to photobleaching as in $\boldsymbol{A}$; see main text). Upon stimulation initiation, the SI decay rate increases. Note that upon stimulation cessation, the SI does not recover to baseline levels (as EGFP:SV2A fluorescence does). Rather, it gradually converges to baseline rates of SI decay. $C$, Distribution of normalized ranges (range/mean) for the nonstimulated dataset, calculated for each bouton as illustrated in Figure 2D. of powerful forces that act to restore SV pool sizes to particular "set points." Finally, they also indicate that such set points are not entirely constant and exhibit activity-independent changes over longer time scales.

\section{Relationships between synaptic activity and active zone remodeling}

What determines the SV pool size set point at a particular synapse? One possibility is that such set points are determined by relatively stable molecular complexes found at active zones (AZs), namely, the cytoskeleton of the active zone (CAZ) (Schoch and Gundelfinger, 2006; Sigrist and Schmitz, 2011). Indeed, there seems to be an excellent correlation between AZ size and total (Yeow and Peterson, 1991; Pierce and Mendell, 1993), docked (Schikorski and Stevens, 1997, 1999; Murthy et al., 2001), and readily releasable (Matz et al., 2010) SV pool sizes. Following this line of reasoning, the gradual convergence of SI decay rates in stimulated preparations to those measured in nonstimulated preparations might indicate that CAZ remodeling is not strongly affected by the activity regimes tested here, and consequently, following the cessation of stimulation, SVs gradually redistribute among synapses until SV pool sizes once again match the sizes of their respective CAZ.

To examine the degree to which CAZ remodeling is affected by the stimulation paradigms used here, we repeated the same experiments described above using GFP:Bsn95-3938, a fusion protein of GFP and a slightly truncated variant of the CAZ molecule Bassoon. GFP:Bsn95-3938 was previously shown to be targeted correctly to presynaptic sites (Dresbach et al., 2003, 2006) and was used to study Bassoon recruitment (Bresler et al., 2004; Bury and Sabo, 2011), Bassoon exchange rates (Tsuriel et al., 2009), and AZ remodeling (Matz et al., 2010). As previously reported in the aforementioned studies, GFP:Bsn95-3938 exhibits a punctate distribution pattern when expressed in cultured hippocampal neurons (Fig. 6A). In relatively mature preparations such as those used here, most puncta correspond to functional presynaptic sites.

Time-lapse recordings, performed at $5 \mathrm{~min}$ intervals as described above, revealed that the fluorescence intensity of individual GFP:Bsn95-3938 puncta changed gradually over time (Fig. $6 B, C$ ) (see also Matz et al., 2010). Examining the normalized ranges of GFP:Bsn95-3938 fluorescence changes revealed that GFP:Bsn95-3938 fluorescence changed considerably in most synapses over this $\sim 3 \mathrm{~h}$ period, with $>59$ and $>20 \%$ of boutons exhibiting changes $>30$ and $50 \%$, respectively (Fig. $6 D$ ).

Stimulating neurons expressing GFP:Bsn95-3938 for $1 \mathrm{~h}$ using the same stimulation paradigm described above $(30 \mathrm{~s}$ at $20 \mathrm{~Hz}$, every $5 \mathrm{~min}$ ) did not lead to detectable changes in GFP:Bsn953938 fluorescence (Fig. 6E). Furthermore, this stimulation paradigm did not accelerate the rate of SI decay compared with that measured in nonstimulated preparations (Fig. $6 F$ ). These findings indicate that CAZ remodeling is not significantly affected by the stimulation paradigms tested here.

GFP:Bsn95-3938 puncta, in common with GFP:SV2A puncta (Figs. 2, 5), exhibited significant activity-independent changes in their fluorescence over time. If SV pool size set points are governed by AZ sizes, spontaneous AZ remodeling might underlie some of the spontaneous changes recorded in total SV pool sizes. We could not rule out the possibility, however, that the extensive GFP:Bsn95-3938 dynamics we observed were, at least partially attributable to GFP:Bsn95-3938 overexpression (Bresler et al., 2004) or the lack of the first 94 aa of native bassoon. To obtain an estimate of inherent CAZ dynamics in a minimally perturbed 

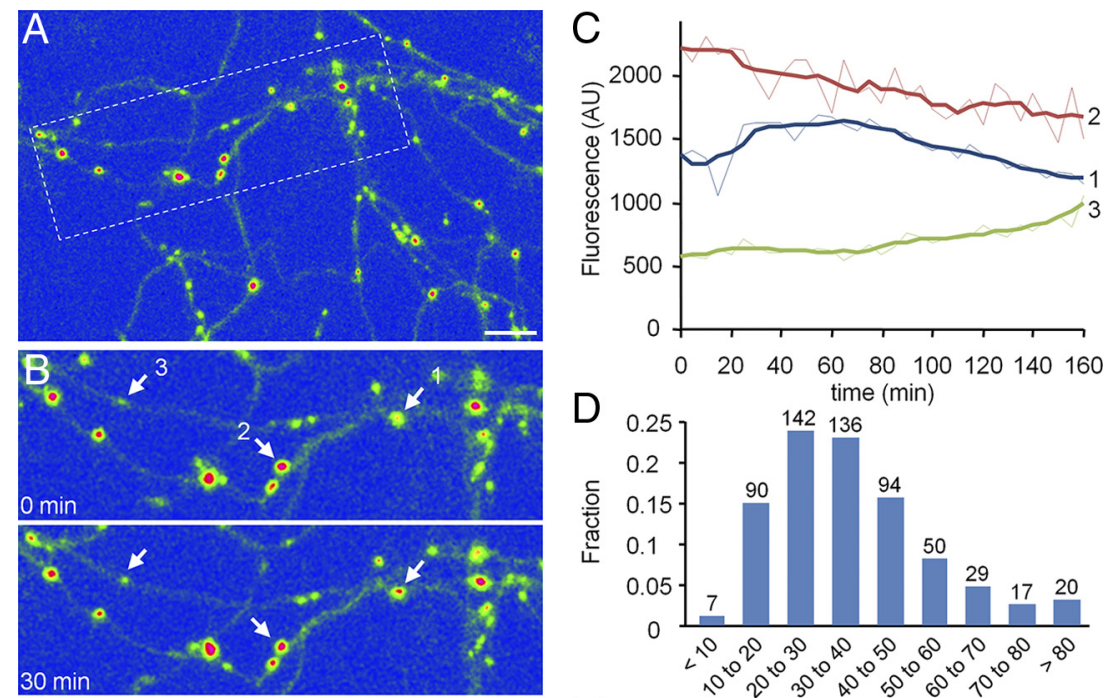

\section{D
든
진}
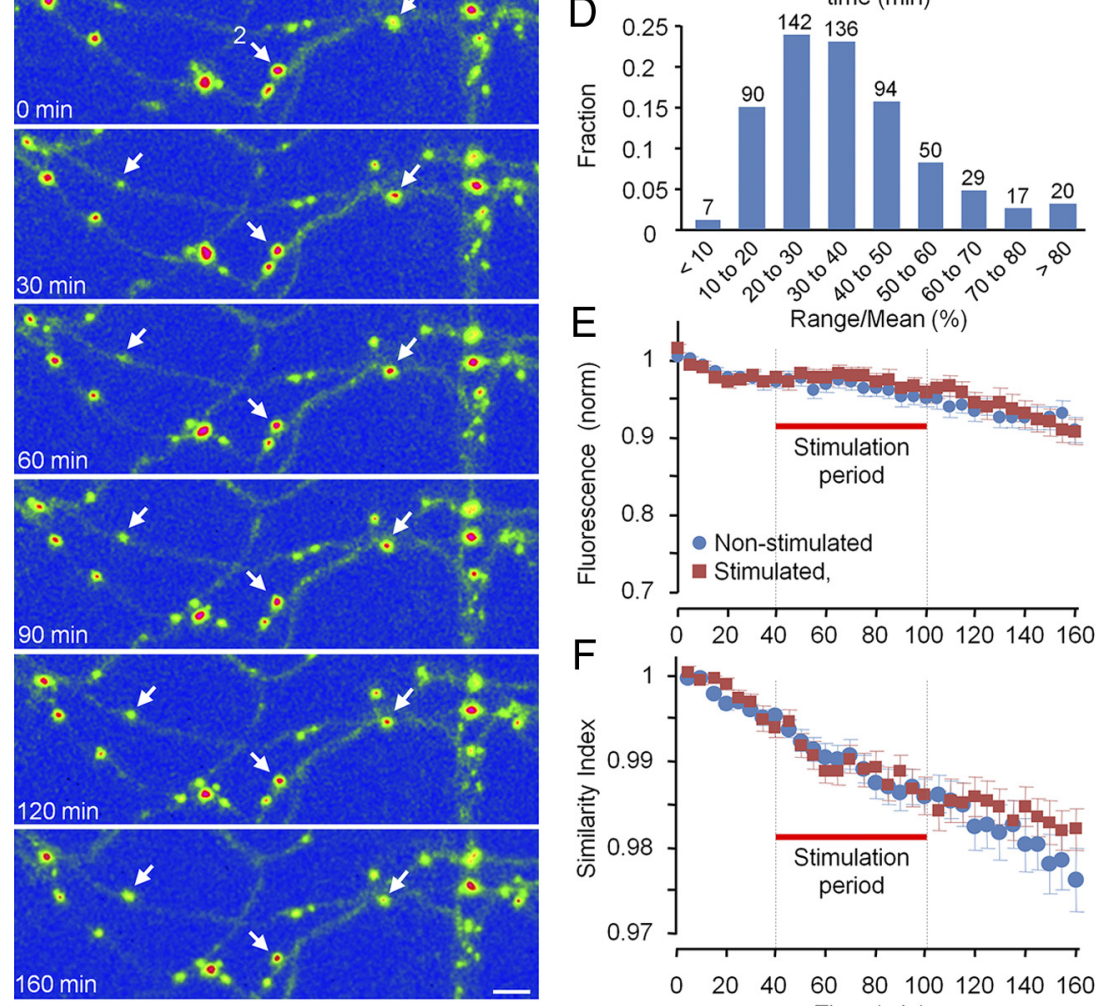

$\mathrm{F}$

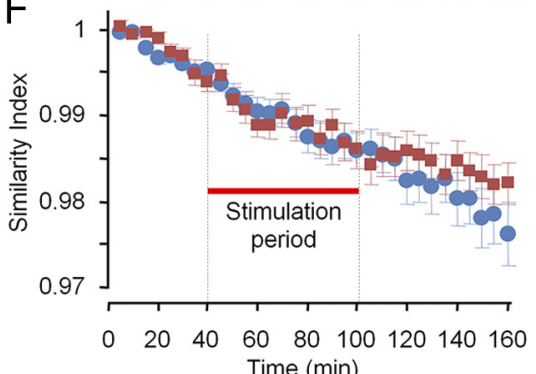

Figure 6. Use dependence of active zone size tenacity. $\boldsymbol{A}$, An axon belonging to a neuron expressing GFP:Bsn95-3938. Fluorescence levels are encoded in false color according to color scale in Figure $2 A$. Scale bar, $10 \mu \mathrm{m}$. B, Time-lapse image of region enclosed in rectangle in $\boldsymbol{A}$. Images were collected at 5 min intervals (only a subset of these are shown here). Scale bar, $5 \mu \mathrm{m}$. $\boldsymbol{C}$, Changes in fluorescence over time for three boutons pointed to by arrows in $\boldsymbol{B}$. Raw measurements depicted by thin lines; smoothed (5-point low-pass filter) depicted as thick lines. $\boldsymbol{D}$, Distribution of normalized range values for all boutons in these experiments (nonstimulated dataset only; see below). $\boldsymbol{E}$, Changes in GFP:Bsn95-3938 fluorescence over time before, during, and after a stimulation period as in Figure 5. Data are shown for two conditions (average \pm SEM): no stimulation (5 experiments, 26 sites, 639 boutons); and stimulation (5 experiments, 32 sites, 866 boutons). Both sets of experiments were performed in the presence of $C N Q X$ and $A P 5$. The gradual reduction in baseline fluorescence $(\sim 10 \%)$ is due to photobleaching. $\boldsymbol{F}, \mathrm{SI}$ decay rates for the same data and conditions as in $\boldsymbol{E}$ (average \pm SEM). Note that stimulation did not affect the SI decay rate. The apparent separation of the curves on the right-hand side is entirely attributable to 2 (of 32) atypical outliers in the stimulated cell dataset and is therefore not significant.

system, we turned to a mutant knock-in mouse line in which the CAZ molecule Munc13-1, fused to EYFP, is expressed from the Munc13-1 genomic locus (Kalla et al., 2006). Munc13-1:EYFPcontaining neurons and synapses are functionally identical to those of wild-type (WT) mice. Furthermore, in mice homozygous for this mutation, Munc13-1:EYFP is the only species of Munc13-1 these mice express. Finally and most importantly, Munc13-1:EYFP expression is controlled by endogenous regulatory systems, and, indeed, its expression levels are no different from those of Munc13-1 in WT mice (Kalla et al., 2006). We thus prepared cortical neuronal cultures from homozygous Munc13-1:EYFP mice and followed Munc13-1:EYFP dynamics in such cultures
(Fig. 7A). Unfortunately, as Munc13-1: EYFP is relatively sensitive to photobleaching (Kalla et al., 2006) collecting images at 5 min intervals (as performed here for SV2: GFP and GFP:Bsn95-3938) resulted in practically useless data due to extensive photobleaching (data not shown). However, judicious sampling at longer intervals matched to fluorescence recovery rates allowed us to follow CAZ dynamics over long time scales in this minimally perturbed system. We thus performed time-lapse imaging of cortical neurons from Munc13-1: EYFP mice (days 20-25 in vitro) at $1 \mathrm{~h}$ intervals for $17-25 \mathrm{~h}$ (Fig. 7B). Under such conditions, photobleaching levels were quite acceptable $(\sim 20 \%$ after $17 \mathrm{~h})$ (Fig. $7 E$ ). As practically all presynaptic boutons are fluorescent in these preparations (unlike the situation in sparsely infected/transfected preparations), we had to limit our analysis to Munc13-1:EYFP puncta that were well separated from their neighbors.

As shown in Figure $7 B-D$, Munc13-1: EYFP puncta exhibited significant changes in their fluorescence, which progressively became more pronounced, with $>70$ and $>23 \%$ of boutons exhibiting changes of $>40$ and $60 \%$, respectively, after periods of $17 \mathrm{~h}$. Moreover, and in common with SV2: GFP and GFP:Bsn95-3938, the similarity index decayed significantly over this time period. Specifically, the average SI at the end of $17 \mathrm{~h}(\sim 0.975)$ was half way between 1.0 and the average SI calculated for shuffled data $(\sim 0.94)$; that is, nearly halfway between the situation in which each bouton perfectly maintained its original Munc13-1: EYFP fluorescence and a situation in which each bouton ended up with a random fluorescence value.

These experiments therefore demonstrate that AZs can undergo significant spontaneous remodeling, which does not seem to be affected by the stimulation paradigms tested here. These findings are therefore congruent with the possibility that the CAZ dictates, to some degree, the SV pool size set point of individual presynaptic boutons (see also Matz et al., 2010).

\section{Discussion}

Here we examined the ability of individual boutons to maintain their SV content, and the degree to which this ability is affected by intense activity. We found that the sizes of total SV pools of individual boutons, belonging to the same axons, gradually change over time scales of several hours, and that these basal changes occur independently of activity. Intermittent stimulation for $1 \mathrm{~h}$ accelerated the rate at which SV pool sizes of nearby boutons changed relative to each other. Following stimulation cessation, vesicle pool size change rates gradually converged to basal change rates. Identical stimulation paradigms did not affect 
the Bassoon content of individual synaptic boutons, yet the synaptic contents of Bassoon, as well as those of Munc-13-1, exhibited significant spontaneous changes over time scales of hours. These findings indicate that enhanced levels of synaptic activity can accelerate the redistribution of SVs among nearby synapses. They also reveal, however, the presence of potent forces that act to restore particular set points in terms of total SV pool sizes. Finally, while these findings are in line with the notion that SV pool size set points are defined by AZ size, they also indicate that neither AZ sizes nor SV pool size set points are particularly stable, questioning the long-term tenacity of individual presynaptic specializations.

\section{Measuring changes in SV pools sizes}

Presynaptic boutons contain large number of SVs, which are traditionally divided into various pools (docked, readily releasable, reserve, functional, and resting) based on their release kinetics and physical location. Our means of measuring SV pool size-EGFP: SV2A fluorescence- does not distinguish between these pools and therefore can only be viewed as a measure of the total SV pool size. It should be noted, however, that this measure is somewhat indirect and might have suffered from some inaccuracies. For example, if EGFP:SV2A copy number differed greatly among individual SVs, changes in total fluorescence could conceivably reflect the reshuffling of SVs with different EGFP:SV2A content among neighboring boutons. Nevertheless, we have good reasons to believe that EGFP:SV2A fluorescence provides a reasonable estimate of total SV pool size. First, we observed a good correlation between FM4-64 uptake and EGFP:SV2A fluorescence (Fig. $1 F$ ). Second, in a recent study that quantified copy number variability of SV proteins within SV populations, SV2 was found to have the most consistent copy number, with nearly all SVs containing five copies of this protein (Mutch et al., 2011). Third, all experiments were performed at least 2 weeks from infection, at which time EGFP:SV2A expression would have been nearly uniform. Finally, as individual boutons contain dozens to hundreds of SVs, EGFP:SV2A copy number variability would become a highly improbable explanation for the large changes in EGFP:SV2A fluorescence recorded at individual boutons (Fig. 5 C). We thus conclude that EGFP:SV2A fluorescence provides reasonable estimates of (total) SV pool sizes.

Relative changes in SV pool sizes were quantified by the SIthe similarity of two sets of fluorescence measurements made from the same puncta at different times. It is important to note that this measure, unlike EGFP:SV2A fluorescence, was not immediately maximally affected by stimulation-induced, rapid dispersion of EGFP:SV2A (Fig. 5, compare A, B). Therefore, the greater SI decay rates during prolonged stimulation signified the gradual redistribution of EGFP:SV2A among nearby synapses, not merely immediate GFP:SV2A dispersion. Similarly, the SI converged to basal SI decay rates slowly and gradually following stimulation cessation, whereas the fluorescence recovery was nearly complete within minutes, further indicating that the SI is not simply a measure of activity-induced SV2A dispersion and recovery.

It is worth re-emphasizing that uncertainties associated with tracking highly dynamic puncta, biased our results toward the relatively stable bouton population. It follows that the dynamics reported here for SV2A (and for the AZ molecules imaged) un- 
doubtedly underestimate the full extent of vesicular mobilization (and AZ remodeling). In principle, kymograph analysis (Fig. 3) should have resolved this problem, as it allowed us to quantify the dynamics of all EGFP:SV2A puncta along axon segments. This form of analysis, however, did not reveal a significant effect of stimulation on SV mobilization rates. Given that this measure was mainly affected by small longitudinal shifts in bouton positions, we suspect that the effects of stimulation might have been washed out by this strong source of profile decorrelation.

A potential concern is the possible effect of SV2A overexpression. While this is always a concern when using XFP-tagged proteins, we do not think this is a major problem here. First, the particular lentiviral construct used here lacks the Woodchuck Hepatitis Virus Posttranscriptional Regulatory Element (Zufferey et al., 1999) usually used to enhance expression levels (Lois et al., 2002), which are typically low even when this element is present. Second, FM4-64 uptake in EGFP:SV2A-positive puncta was no greater than that observed in neighboring uninfected neurons. As FM4-64 uptake and EGFP:SV2A content were strongly correlated (Fig. $1 F$ ), the absence of excessive FM4-64 uptake in EGFP:SV2A-positive boutons argues against significant SV2A overexpression. Finally, it is worth mentioning that we used an extremely low viral titer, resulting in very sparse infection, practically guaranteeing that all axonal segments in a field of view originated from only one neuron in the majority of cases, and at most from two to three neurons.

\section{Use dependence of presynaptic tenacity}

Our findings indicate that continuous activity accelerates the rates at which SV contents of individual synapses change over time. Yet, the gradual convergence of SI decay rates in stimulated preparations with those measured in nonstimulated cells, and the observation that SI values never decayed all the way down to the values that would be measured if all relationships between initial and final pools sizes were lost, point to the existence of potent mechanisms that enable presynaptic boutons to (at least partially) conserve their SV pool sizes in face of intersynaptic and intrasynaptic vesicle trafficking. What might these be? In all likelihood, these are the same mechanisms that act to confine SVs to presynaptic boutons during synaptogenesis and later on. While not yet entirely understood, they probably involve, among many others, actin (Cingolani and Goda, 2008), Synapsins (Cesca et al., 2010), trans-synaptic complexes (Linhoff et al., 2009; Brigidi and Bamji, 2011), efficient endocytosis mechanisms (Kamin et al., 2010), as well as particular protein kinases and phosphatases (Lee et al., 2008; Kim and Ryan, 2010; reviewed in Pechstein and Shupliakov, 2010; Waites and Garner, 2011). Whatever these mechanisms might be, our findings indicate that they collectively act to restore synapse-specific set points.

What, however, determines the set points for particular synapses? There are good reasons to believe that such set points are dictated, at least in part, by AZ size, or, more specifically, by CAZ size. As mentioned above, strong relationships between $\mathrm{AZ}$ and various SV pool sizes have been reported (Yeow and Peterson, 1991; Pierce and Mendell, 1993; Schikorski and Stevens, 1997, 1999; Murthy et al., 2001). Our findings that prolonged stimulation paradigms do not affect the synaptic contents of Bassoon are in line with this idea, as they indicate that the AZ, by remaining "indifferent" to the intense dynamics associated with SV recycling, represents a relatively stable nucleus that could impose a particular set point in terms of SV pool size. Indeed, the molecular dynamics of major CAZ components (Munc 13-1, Piccolo, Bassoon) are not strongly affected by acute stimulation (Kalla et al., 2006; Tao-Cheng, 2006; Tsuriel et al., 2009), whereas the elimination of Piccolo is associated with deeper activity-induced dispersion and slower reaccumulation of Synapsin (Leal-Ortiz et al., 2008) probably due to Piccolo-related regulation of actin dynamics (Waites et al., 2011). In this regard, a recent study by Matz et al. (2010) is of particular interest as it reported that spontaneous changes in $\mathrm{AZ}$ size correlate well with changes in readily releasable SV pool sizes, lending further credence to the idea that SV content set points are largely determined by CAZ properties.

Whereas our data indicate that presynaptic sites possess "restorative" forces that allow them to maintain their SV pools, they also indicate that over longer time scales neither SV pool sizes nor AZ sizes are particularly stable. While some of these dynamics might be attributable to fusion protein overexpression (in particular where Bassoon is concerned), we observed similar dynamics in neurons obtained from Munc13-1:YFP mice, which do not overexpress this fusion protein (Kalla et al., 2006). In fact, the dynamics measured for EGFP:SV2A and Munc13-1:YFP were remarkably similar (compare Figs. $5 B, C, 7 D, F$ ). The dynamics we observed here are probably exaggerated compared with those occurring in the adult brain, not only because of cell culture issues but also because the somewhat immature developmental status of our preparations. Therefore, it is likely that the phenomena reported here, if they occur in vivo, occur on slower time scales. Nevertheless, given the remarkably similar conclusions of prior in vitro and in vivo studies concerning synaptic tenacity (for example, Tsuriel et al., 2006 and Grey et al., 2006; Minerbi et al., 2009 and Loewenstein et al., 2011), we carefully conclude that presynaptic tenacity, and indeed synaptic tenacity in general might be inherently limited.

\section{References}

Ahmari SE, Buchanan J, Smith SJ (2000) Assembly of presynaptic active zones from cytoplasmic transport packets. Nat Neurosci 3:445-451.

Bajjalieh SM, Peterson K, Shinghal R, Scheller RH (1992) SV2, a brain synaptic vesicle protein homologous to bacterial transporters. Science 257:1271-1273.

Bresler T, Shapira M, Boeckers T, Dresbach T, Futter M, Garner CC, Rosenblum K, Gundelfinger ED, Ziv NE (2004) Postsynaptic density assembly is fundamentally different from presynaptic active zone assembly. J Neurosci 24:1507-1520.

Brigidi GS, Bamji SX (2011) Cadherin-catenin adhesion complexes at the synapse. Curr Opin Neurobiol 21:208-214.

Bury LA, Sabo SL (2011) Coordinated trafficking of synaptic vesicle and active zone proteins prior to synapse formation. Neural Dev 6:24.

Cesca F, Baldelli P, Valtorta F, Benfenati F (2010) The synapsins: key actors of synapse function and plasticity. Prog Neurobiol 91:313-348.

Chi P, Greengard P, Ryan TA (2001) Synapsin dispersion and reclustering during synaptic activity. Nat Neurosci 4:1187-1193.

Chi P, Greengard P, Ryan TA (2003) Synaptic vesicle mobilization is regulated by distinct synapsin I phosphorylation pathways at different frequencies. Neuron 38:69-78.

Cingolani LA, Goda Y (2008) Actin in action: the interplay between the actin cytoskeleton and synaptic efficacy. Nat Rev Neurosci 9:344-356.

Colicos MA, Collins BE, Sailor MJ, Goda Y (2001) Remodeling of synaptic actin induced by photoconductive stimulation. Cell 107:605-616.

Dai Z, Peng HB (1996) Dynamics of synaptic vesicles in cultured spinal cord neurons in relationship to synaptogenesis. Mol Cell Neurosci 7:443-452.

Darcy KJ, Staras K, Collinson LM, Goda Y (2006) Constitutive sharing of recycling synaptic vesicles between presynaptic boutons. Nat Neurosci 9:315-321.

De Paola V, Arber S, Caroni P (2003) AMPA receptors regulate dynamic equilibrium of presynaptic terminals in mature hippocampal networks. Nat Neurosci 6:491-500.

Dresbach T, Hempelmann A, Spilker C, tom Dieck S, Altrock WD, Zuschratter W, Garner CC, Gundelfinger ED (2003) Functional regions of the presynaptic cytomatrix protein bassoon: significance for synaptic targeting and cytomatrix anchoring. Mol Cell Neurosci 23:279-291.

Dresbach T, Torres V, Wittenmayer N, Altrock WD, Zamorano P, Zuschrat- 
ter W, Nawrotzki R, Ziv NE, Garner CC, Gundelfinger ED (2006) Assembly of active zone precursor vesicles: obligatory trafficking of presynaptic cytomatrix proteins Bassoon and Piccolo via a trans-Golgi compartment. J Biol Chem 281:6038-6047.

Feany MB, Lee S, Edwards RH, Buckley KM (1992) The synaptic vesicle protein SV2 is a novel type of transmembrane transporter. Cell 70:861-867.

Fernandez-Alfonso T, Ryan TA (2008) A heterogeneous "resting" pool of synaptic vesicles that is dynamically interchanged across boutons in mammalian CNS synapses. Brain Cell Biol 36:87-100.

Fernández-Alfonso T, Kwan R, Ryan TA (2006) Synaptic vesicles interchange their membrane proteins with a large surface reservoir during recycling. Neuron 51:179-186.

Gal A, Eytan D, Wallach A, Sandler M, Schiller J, Marom S (2010) Dynamics of excitability over extended timescales in cultured cortical neurons. J Neurosci 30:16332-16342.

Gray NW, Weimer RM, Bureau I, Svoboda K (2006) Rapid redistribution of synaptic PSD-95 in the neocortex in vivo. PLoS Biol 4:e370.

Hellwig B, Schüz A, Aertsen A (1994) Synapses on axon collaterals of pyramidal cells are spaced at random intervals: a Golgi study in the mouse cerebral cortex. Biol Cybern 71:1-12.

Hopf FW, Waters J, Mehta S, Smith SJ (2002) Stability and plasticity of developing synapses in hippocampal neuronal cultures. J Neurosci 22:775-781.

Kalla S, Stern M, Basu J, Varoqueaux F, Reim K, Rosenmund C, Ziv NE, Brose N (2006) Molecular dynamics of a presynaptic active zone protein studied in Munc13-1-enhanced yellow fluorescent protein knock-in mutant mice. J Neurosci 26:13054-13066.

Kamin D, Lauterbach MA, Westphal V, Keller J, Schönle A, Hell SW, Rizzoli SO (2010) High- and low-mobility stages in the synaptic vesicle cycle. Biophys J 99:675-684.

Kim SH, Ryan TA (2010) CDK5 serves as a major control point in neurotransmitter release. Neuron 67:797-809.

Kraszewski K, Mundigl O, Daniell L, Verderio C, Matteoli M, De Camilli P (1995) Synaptic vesicle dynamics in living cultured hippocampal neurons visualized with CY3-conjugated antibodies directed against the lumenal domain of synaptotagmin. J Neurosci 15:4328-4342.

Krueger SR, Kolar A, Fitzsimonds RM (2003) The presynaptic release apparatus is functional in the absence of dendritic contact and highly mobile within isolated axons. Neuron 40:945-957.

Leal-Ortiz S, Waites CL, Terry-Lorenzo R, Zamorano P, Gundelfinger ED, Garner CC (2008) Piccolo modulation of Synapsinla dynamics regulates synaptic vesicle exocytosis. J Cell Biol 181:831-846.

Lee SH, Peng IF, Ng YG, Yanagisawa M, Bamji SX, Elia LP, Balsamo J, Lilien J, Anastasiadis PZ, Ullian EM, Reichardt LF (2008) Synapses are regulated by the cytoplasmic tyrosine kinase Fer in a pathway mediated by p120catenin, Fer, SHP-2, and beta-catenin. J Cell Biol 183:893-908.

Li Z, Murthy VN (2001) Visualizing postendocytic traffic of synaptic vesicles at hippocampal synapses. Neuron 31:593-605.

Linhoff MW, Laurén J, Cassidy RM, Dobie FA, Takahashi H, Nygaard HB, Airaksinen MS, Strittmatter SM, Craig AM (2009) An unbiased expression screen for synaptogenic proteins identifies the LRRTM protein family as synaptic organizers. Neuron 61:734-749.

Loewenstein Y, Kuras A, Rumpel S (2011) Multiplicative dynamics underlie the emergence of the log-normal distribution of spine sizes in the neocortex in vivo. J Neurosci 31:9481-9488.

Lois C, Hong EJ, Pease S, Brown EJ, Baltimore D (2002) Germline transmission and tissue-specific expression of transgenes delivered by lentiviral vectors. Science 295:868-872.

Lucido AL, Suarez Sanchez F, Thostrup P, Kwiatkowski AV, Leal-Ortiz S, Gopalakrishnan G, Liazoghli D, Belkaid W, Lennox RB, Grutter P, Garner CC, Colman DR (2009) Rapid assembly of functional presynaptic boutons triggered by adhesive contacts. J Neurosci 29:12449-12466.

Matz J, Gilyan A, Kolar A, McCarvill T, Krueger SR (2010) Rapid structural alterations of the active zone lead to sustained changes in neurotransmitter release. Proc Natl Acad Sci U S A 107:8836-8841.

Minerbi A, Kahana R, Goldfeld L, Kaufman M, Marom S, Ziv NE (2009) Long-term relationships between synaptic tenacity, synaptic remodeling, and network activity. PLoS Biol 7:e1000136.

Murthy VN, Sejnowski TJ, Stevens CF (1997) Heterogeneous release properties of visualized individual hippocampal synapses. Neuron 18:599-612.

Murthy VN, Schikorski T, Stevens CF, Zhu Y (2001) Inactivity produces increases in neurotransmitter release and synapse size. Neuron 32:673-682.
Mutch SA, Kensel-Hammes P, Gadd JC, Fujimoto BS, Allen RW, Schiro PG, Lorenz RM, Kuyper CL, Kuo JS, Bajjalieh SM, Chiu DT (2011) Protein quantification at the single vesicle level reveals that a subset of synaptic vesicle proteins are trafficked with high precision. J Neurosci 31:1461-1470.

Nowack A, Yao J, Custer KL, Bajjalieh SM (2010) SV2 regulates neurotransmitter release via multiple mechanisms. Am J Physiol Cell Physiol 299:C960-C967.

Pechstein A, Shupliakov O (2010) Taking a back seat: synaptic vesicle clustering in presynaptic terminals. Front Syn Neurosci 2:143.

fPierce JP, Mendell LM (1993) Quantitative ultrastructure of Ia boutons in the ventral horn: scaling and positional relationships. J Neurosci 13:4748-4763.

Sankaranarayanan S, Ryan TA (2000) Real-time measurements of vesicleSNARE recycling in synapses of the central nervous system. Nat Cell Biol 2:197-204.

Sasaki T, Matsuki N, Ikegaya Y (2007) Metastability of active CA3 networks. J Neurosci 27:517-528.

Schikorski T, Stevens CF (1997) Quantitative ultrastructural analysis of hippocampal excitatory synapses. J Neurosci 17:5858-5867.

Schikorski T, Stevens CF (1999) Quantitative fine-structural analysis of olfactory cortical synapses. Proc Natl Acad Sci U S A 96:4107-4112.

Schoch S, Gundelfinger ED (2006) Molecular organization of the presynaptic active zone. Cell Tissue Res 326:379-391.

Shepherd GM, Harris KM (1998) Three-dimensional structure and composition of CA33CA1 axons in rat hippocampal slices: implications for presynaptic connectivity and compartmentalization. J Neurosci 18:8300-8310.

Shepherd GM, Raastad M, Andersen P (2002) General and variable features of varicosity spacing along unmyelinated axons in the hippocampus and cerebellum. Proc Natl Acad Sci U S A 99:6340-6345.

Sigrist SJ, Schmitz D (2011) Structural and functional plasticity of the cytoplasmic active zone. Curr Opin Neurobiol 21:144-150.

Star EN, Newton AJ, Murthy VN (2005) Real-time imaging of Rab3a and Rab5a reveals differential roles in presynaptic function. J Physiol 569:103-117.

Staras K (2007) Share and share alike: trading of presynaptic elements between central synapses. Trends Neurosci 30:292-298.

Staras K, Branco T (2010) Sharing vesicles between central presynaptic terminals: implications for synaptic function. Front Synaptic Neurosci 2:20.

Staras K, Branco T, Burden JJ, Pozo K, Darcy K, Marra V, Ratnayaka A, Goda Y (2010) A vesicle superpool spans multiple presynaptic terminals in hippocampal neurons. Neuron 66:37-44.

Tao-Cheng JH (2006) Activity-related redistribution of presynaptic proteins at the active zone. Neuroscience 141:1217-1224.

Tao-Cheng JH, Dosemeci A, Winters CA, Reese TS (2006) Changes in the distribution of calcium calmodulin-dependent protein kinase II at the presynaptic bouton after depolarization. Brain Cell Biol 35:117-124.

Tsuriel S, Geva R, Zamorano P, Dresbach T, Boeckers T, Gundelfinger ED, Garner CC, Ziv NE (2006) Local sharing as a predominant determinant of synaptic matrix molecular dynamics. PLoS Biol 4:e271.

Tsuriel S, Fisher A, Wittenmayer N, Dresbach T, Garner CC, Ziv NE (2009) Exchange and redistribution dynamics of the cytoskeleton of the active zone molecule bassoon. J Neurosci 29:351-358.

Wagenaar DA, Pine J, Potter SM (2006) Searching for plasticity in dissociated cortical cultures on multi-electrode arrays. J Negat Results Biomed 5:16.

Waites CL, Garner CC (2011) Presynaptic function in health and disease. Trends Neurosci 34:326-337.

Waites CL, Leal-Ortiz SA, Andlauer TF, Sigrist SJ, Garner CC (2011) Piccolo regulates the dynamic assembly of presynaptic F-actin. J Neurosci 31:14250-14263.

Waters J, Smith SJ (2002) Vesicle pool partitioning influences presynaptic diversity and weighting in rat hippocampal synapses. J Physiol 541:811-823.

Westphal V, Rizzoli SO, Lauterbach MA, Kamin D, Jahn R, Hell SW (2008) Video-rate far-field optical nanoscopy dissects synaptic vesicle movement. Science 320:246-249.

Wienisch M, Klingauf J (2006) Vesicular proteins exocytosed and subsequently retrieved by compensatory endocytosis are nonidentical. Nat Neurosci 9:1019-1027.

Yeow MB, Peterson EH (1991) Active zone organization and vesicle content scale with bouton size at a vertebrate central synapse. J Comp Neurol 307:475-486.

Zufferey R, Donello JE, Trono D, Hope TJ (1999) Woodchuck hepatitis virus posttranscriptional regulatory element enhances expression of transgenes delivered by retroviral vectors. J Virol 73:2886-2892. 